\title{
Vornamenwahl in einem deutsch-rumänischen Umfeld zwischen regionalen Gepflogenheiten und europäischen Trends
}

\section{Gerhild RUDOLF}

Drd., Lucian-Blaga-Universität in Sibiu/Hermannstadt; E-Mail: rudolfgerhild@gmail.com

\begin{abstract}
An essential factor for the naming practice lies in the language(s) spoken by that certain family. In the nowadays very common multilingual families in Transylvania, the so called ,mixed marriages', the linguistic contact also becomes manifest in the field of onomatology. Out of the vast subject matter, four aspects will be approached: the decline of the tradition of naming a child after a parent; naming practices following ethnic reasons in order to denote a certain identity; naming preferences for international names in mixed families; the increasing diversification and interculturality of name-giving due to globalization and the impact of social media. Concrete examples - based on baptismal registers of the local Lutheran Church - illustrate the monitored trends.
\end{abstract}

Key words: onomatology,forenames, naming practices, Transylvanian Saxons, German, Romanian, baptismal register, mixed marriages, linguistic contact, hybridisation.

In der multiethnischen und multilingualen Region Siebenbürgen ist auch eine Vielfalt an Vornamen festzustellen, was Untersuchungen zur Onomastik besonders reizvoll macht. Phänomene des Sprach- und Kulturkontakts sind an Personennamen ablesbar. Die Entscheidung der Eltern, welchen Vornamen sie ihrem Kind geben, hängt von vielen Faktoren, beispielsweise von ethnischen, religiös-konfessionellen und familiären Beweggründen ab. Die Motive der Namenwahl korrelieren zudem mit dem 
Bildungsniveau und dem Zeitgeist. In der Namenwahl lassen sich Muster entdecken, die sich von Generation zu Generation wandeln. Traditionelle Namen weichen modischen, werden aber später wieder recht beliebt. Zudem sind auch sozio-onomastische ${ }^{1}$ Unterschiede feststellbar, beruhend auf (sich heutzutage zunehmend angleichenden) unterschiedlichen Gepflogenheiten auf dem Land und in der Stadt.

Ein wesentlicher Faktor für die Vornamenwahl ist die Sprache der Familie. Im Falle der in Siebenbürgen inzwischen häufig auftretenden sprachverschiedenen Familien (in den so genannten Mischehen) handelt es sich gleich um zwei oder mehr Sprachen, die berücksichtigt werden, und folglich tritt der Sprachkontakt auch in der Onomastik in Erscheinung.

Aus der Fülle der Thematik werden vier besondere Aspekte untersucht: die lange Beharrung auf Traditionsnamen; Namenwahl aus ethnischen Gründen, um die Identität zu markieren; Präferenz für internationale Namen in mehrsprachigen Familien; die zunehmende Diversifizierung und Interkulturalität in der Namensgebung als Folge der Globalisierung und der sozialen Medien.

Der Fokus der kurzen Betrachtung im Bereich der Interferenz-Onomastik ist auf deutsche und deutsch-rumänische Sprachmilieus in Siebenbürgen gerichtet und umfasst anhand von Stichproben die Zeitspanne der letzten 100 Jahre. Konkrete Beispiele - basierend auf Hermannstädter kirchlich-evangelischen Taufregistern der Jahre 1910 (in Ungarn), 1940 (im

1 Die Sozio-Onomastik ist ein Bereich der Namensforschung. Laut Löfflererforschtdie Sozio-Onomastik, ,die sozialeZuordnungsfunktion von Personennamen oder die gruppenspezifischen Motive der Namensgebung. Selbst die Institution der Personennamengebung in ihrem privat-arbiträren und dem öffentlich-administrativen Teil ist eine höchst bedeutende Kontaktzone von Sprache und Gesellschaft. Sozio-Onomastik im engeren Sinn befasst sich mit der sozialen Markiertheit von Personennamen." Löffler, Heinrich: Germanistische Soziolinguistik. 4., neu bearb. Aufl., Berlin: Erich Schmidt Verlag, 2010, S. 165. 
Königreich Rumänien), 1970 (in der Sozialistischen Republik Rumänien), 2000 (in Rumänien) und 2017 (in Rumänien/EUMitgliedsstaat) - veranschaulichen die beobachteten Trends. Taufregister geben Auskunft über die Namen eines Jahrgangs innerhalb einer Konfession, sofern es sich um die Taufen Neugeborener handelt, wie es in der Evangelischen Kirche A. B. üblich ist. Die Namensgebung im Falle der genannten Jahrgänge kann auch deshalb aus den Taufregistern erfasst werden, da bis auf sehr vereinzelte Ausnahmen - in der sächsischen Gemeinschaft alle Kinder getauft wurden.

$\mathrm{Da}$ es sich in diesem Beitrag nur um eine erste Annäherung an das Thema handelt, war es nicht möglich, eine akribische wissenschaftliche Analyse zu erarbeiten, die alle möglichen Parameter beachtet. Das untersuchte Korpus von Kirchenbüchern erlaubt einige Schlussfolgerungen, die aber nur im Rahmen einer größeren Arbeit erweitert, verfeinert und vertieft werden könnten. Die bisher gewonnenen Erkenntnisse stehen unter den Stichwörtern: Konservierung, Identitätsmarkierung, Hybridisierung und Diversifizierung.

\section{Konservierung: Familiale Nachbenennung in geschlossener Gemeinschaft}

Die Entwicklung der Vornamen im Sprach- und Schicksalskollektiv der Siebenbürger Sachsen gleicht in großen Zügen der Entwicklung im binnendeutschen Sprachraum, wobei aber einige Verzögerungen und auch einige Sonderentwicklungen festzustellen sind. Eine Verzögerung bezieht sich auf den in Siebenbürgen besonders lange erhaltenen Brauch der Nachbenennung.

Die Nachbenennung war allgemein verbreitet, in den Dorfgemeinden jedoch war sie sogar die Regel. Das heißt, der Name der Eltern wurde an die nächste Generation weitergegeben. Die erstgeborene Tochter erhielt der Vornamen der Mutter, 
der älteste Sohn den des Vaters. Weitere Geschwister erhielten Vornamen der Großeltern und anderer naher Verwandter. Das führte dazu, dass in einem Dorf einige Namen überaus häufig vorkamen. Die Soziologen Renate Weber und Georg Weber zeigen das mustergültig für die Gemeinde Zendersch (Senereuș) auf, wo im Jahr 1944 ,nur 11,5 \% der männlichen und 12,4\% der weiblichen Bevölkerung einen anderen als die vier bzw. drei häufigsten (...) Rufnamen trugen“2. In Zendersch hießen demnach damals von den Siebenbürger Sachsen mehr als dreiviertel der Männer entweder Georg, Andreas, Michael oder Johann und mehr als dreiviertel der Frauen hießen entweder Katharina, Sara oder Anna. Träger seltener Namen wie Heinrich, Helmut, Christine oder Hilda, ,waren meist Angehörige von zugezogenen Handwerkern" ${ }^{\text {3 }}$ oder Beamten.

Die Tradition der familialen Nachbenennung hielt sich bei den Siebenbürger Sachsen länger als in Deutschland. Die von R. und G. Weber genannten Statistiken ${ }^{4}$ belegen für das Jahr 1944 noch vorwiegend das schmale Repertoire der altbewährten Namen, während für Deutschland in derselben Zeit bereits das Abklingen der Nachbenennung festgestellt wurde. ${ }^{5}$

These 1: Die Nachbenennung geht zurück, das Namensrepertoire lässt Muster erkennen.

Statistische Daten zu evangelischen Taufen in Hermannstadt 1910 und 1940:

Im Jahr 1910 gab es in der Hermannstädter Evangelischen Kirchengemeinde A. B. 315 Taufen. 46mal wurde ein Sohn nach dem Vater genannt, 54mal wurde eine Tochter nach der Mutter genannt, einmal erhielt der Sohn den Namen nach der Mutter (Emil/Emilie), zweimal erhielt die Tochter den Namen nach

2 Weber, Georg; Weber, Renate: Zendersch. Eine siebenbürgische Gemeinde im Wandel. München: Delp, 1985, S. 414.

3 Ebenda, S. 411.

4 Siehe Weber, 1985, S. 412.

5 Vgl. Nübling, Damaris: Namen. Eine Einführung in die Onomastik. Tübingen: Narr, 2012, S. 117. 
dem Vater (Henriette/Heinrich). Das ergibt 102 Nachbenennungen bei 315 Taufen - also ein Drittel. ${ }^{6}$

Die beliebtesten Jungennamen waren: Karl, Wilhelm, Friedrich, Gustav, Ernst, Albert, Julius, Otto u. a. Die beliebtesten Mädchennamen waren: Emma, Berta, Hermine, Mathilde, Wilhelmine, Margarete, Frida, Luise, Dorothea u. a.

Unter den Eltern der 1910 Getauften gab es etwa zehn Prozent konfessionell gemischte Ehen zwischen evangelischen und römisch-katholischen Partnern (mit deutschen Namen), und vereinzelt mit Anderskonfessionellen. Gar nicht so selten waren uneheliche Kinder.

Im Jahr 1940 gab es in der Hermannstädter Evangelischen Kirchengemeinde A. B. 335 Taufen. 67mal wurde ein Sohn nach dem Vater genannt, 30mal wurde eine Tochter nach der Mutter genannt. Das ergibt 97 Nachbenennungen bei 335 Taufen - also wieder fast ein Drittel. Weil aber der vom Elternteil übertragene Vorname in gut einer Hälfte der Fälle nur als Zweitname (und somit nicht als Rufname) steht, kann doch behauptet werden, dass die Nachbenennung verglichen zu 1910 abgenommen hat.

Was sich im Vergleich zu einer Generation davor ebenfalls geändert hat, sind die Lieblingsnamen. Die Vielfalt der germanischen Namen hat sichtbar zugenommen, das Repertoire hat sich insgesamt erweitert. Die beliebtesten Jungennamen im Jahr 1940 waren: Horst (Spitzenreiter), Günter, Dieter, Klaus, Kurt, Gerhard, Helmut, Karlheinz, Roland, Siegfried, Werner u. a. Die beliebtesten Mädchennamen waren: Erika (Spitzenreiter), Marianne, Annemarie, Ilse, Karin, Renate, Hildegard, Gertrud, Brigitte, Christa, Herta, u.a. Die meisten Kinder bekamen zwei Vornamen.

Es gab im Jahr 1940 kaum Ehen zwischen Evangelischen und Anderskonfessionellen.

6 Dabei ist nicht zu eruieren, ob das getaufte Kind das erste ist, oder ob es bereits ältere Geschwister hat, bei denen die Nachbenennung vorkommt. Die Namen der Paten und der Großeltern wurden ebenfalls nicht berücksichtigt. 


\section{Identitätsmarkierung: Namenwahl aus ethnischen und kulturellen Gründen}

In der so genannten ,ungarischen Zeit', also vom ÖsterreichUngarischen Ausgleich von 1867 bis zum Ende des Ersten Weltkrieges 1918, wurden die nichtungarischen Bewohner des Landes in Urkunden ${ }^{7}$ oft mit magyarisiertem Namen geführt. Auch die sächsischen evangelischen Pfarrämter waren zeitweise dazu verpflichtet, beim Ausstellen von Akten das ungarische Äquivalent der Vornamen zu verwenden, also beispielsweise János statt Johann, Vilmos statt Wilhelm und Erzsébet statt Elisabeth, Zsófia statt Sophie. Diese diskriminierende Anwendung der interlingualen Allonymie sorgte bei den Minderheiten für Unzufriedenheit. Die verschiedensprachigen Formen für denselben Namen sind im Sinne des Namensträgers nicht einfach austauschbar. ${ }^{8}$

Ähnliche Erfahrungen machten die Siebenbürger Sachsen auch nach der Eingliederung Siebenbürgens in das Königreich Rumänien. Die rumänischen Standesbeamten schrieben die geläufigen biblischen und Heiligennamen gern in der rumänischen Form. So wurde aus Johann ein Ioan, aus Andreas ein Andrei, Katharina wurde zur Ecaterina und Elisabeth zur Elisabeta (oder Elisaveta).

Ein weiteres Problem war (und ist!) auf den Standesämtern die ungenaue Schreibweise in Geburtsurkunden: Rosemarie als

7 Für Personenstandsfragen waren ab 1895 nicht mehr die Kirchen, sondern die Standesämter zuständig.

8 An den Personennamen scheint auf, dass es sich hier um einen sogenannten Hotspot (Begriff, den Hans Jürgen Heringer verwendet) oder Rich Point (ein Konzept, das Michael Agar in Language Shock. Understanding the Culture of Conversation. New York 1994, S. 100. entwickelt hat) handelt, an dem aufgrund kultureller Unterschiede Kommunikationsprobleme entstehen können. Vgl. Heringer, Hans Jürgen: Interkulturelle Kommunikation. 4. Aufl. Tübingen: A. Francke Verlag, 2014. S. 165-169. 
,Rozemari ' geschrieben, Kilian als ,Chilian ' etc. ${ }^{9}$ Die Betroffenen müssen ihr Leben lang in allen offiziellen Papieren diese falsche Schreibweise verwenden - oder aber sich der teuren Mühe unterziehen, ihre sämtlichen Urkunden ändern zu lassen.

Einen typisch rumänischen Namen würden Siebenbürger Sachsen für ihre Kinder nicht wählen (etwa Dragoș, Bogdan, Rodica, Luminița), aber auch Namen, die deutsch und rumänisch gleich oder ähnlich klingen, wurden vermieden. Es mag als ein Zeichen der Assimilationsverweigerung gedeutet werden. ${ }^{10}$

Im deutschsprachigen Gebiet waren die altdeutschen Namen durch die Christianisierung, den katholischen Heiligenkult und die Vorliebe der Reformation für biblische Namen durch Jahrhunderte hindurch in den Hintergrund gedrängt worden. Im 19. Jahrhundert kamen durch die Romantik und andere kulturelle Strömungen altdeutsche Namen wieder stärker in Gebrauch. Der Nationalsozialismus, mit seiner ostentativen und exzessiven Betonung alles Germanischen, förderte diese Entwicklung zusätzlich. Als dann nach dem Zweiten Weltkrieg die alten deutschen Namen in Deutschland spürbar aus der Mode kamen, wurden sie in Siebenbürgen trotzdem gern gegeben, und zwar nicht aus einer etwaigen NS-Nostalgie, sondern aus ethnischkulturellen Motiven. Diese Gepflogenheit ist anhand der evangelischen Taufmatrikeln nachweisbar und betrifft insbesondere die Zeit der kommunistischen Diktatur in Rumänien.

Viele Angehörige der deutschen Minderheit in Rumänien wählten in den 1950er bis 1980er Jahren für ihre

9 Die Beispiele sind aus dem persönlichen Umfeld. Bekannt sind fehlerhafte Schreibweisen sogar bei Familiennamen (Z. B. ,Iohannis' statt Johannis, ,Iunesch'statt Junesch. etc.)

${ }^{10}$ Eine maßgebliche Studie zu den Identitätsbewahrungsstrategien der Siebenbürger Sachsen im sozialistischen Rumänien verfasste Marilyn McArthur 1981; die deutsche Fassung erschien 1990. (McArthur, Marilyn: Zum Identitätswandel der Siebenbürger Sachsen. Eine kulturanthropologische Studie. G. Weber, Hrsg. \& U. Maurer, Übers. Köln, Wien: Böhlau, 1990) 
Kinder germanische Namen mit der Absicht, eine mögliche Rumänisierung des Vornamens zu vermeiden und um die Zugehörigkeit zum deutschen Kulturkreis sichtbar zu machen. Zusätzlich empfand man den Zusammenklang von deutschem Vor- und Nachnamen passend. ${ }^{11}$

In einem rumänischsprachigem Umfeld einen deutschen Namen zu tragen hat einige Folgen: Behörden, Nachbarn, Arbeitskollegen usw. kennen den Namen nicht und können ihn womöglich auch nicht richtig aussprechen und schreiben. Der Namensträger muss seinen Namen oft buchstabieren. Da es für Nichtsprecher des Deutschen schwer zu erkennen ist, dass es sich beispielsweise bei Wiltrud, Dietgund, Ortrun um weibliche Personennamen handelt, kommt es für die betreffenden Namensträgerinnen immer wieder zu Missverständnissen.

These 2: Deutschsprachige Familien im kommunistischen Rumänien wählten häufig altdeutsche Namen.

Statistische Daten zu Vornamen in deutschsprachigen Familien, 1970: Aus dem Taufregister des Jahres 1970 der Evangelischen Kirchengemeinde A. B. in Hermannstadt - mit 363 Taufen - geht hervor, dass alle deutschsprachigen Elternpaare (wo beide Eltern evangelisch sind und deutsche Namen tragen) für ihre Kinder solche Namen ausgesucht haben, die im Deutschen üblich sind und die (im Kontrast zum rumänischen Umfeld) als deutsche Namen erkennbar sind. Darunter sind auch viele altdeutsche (in Deutschland bereits unmoderne) Namen.

Die Hitliste in Hermannstadt führen im Jahr 1970 folgende Namen an: Dieter, Jürgen, Christian (meist mit Zusatznamen), Uwe, Harald, Klaus, Udo, Ingo, Bernd, Werner, Hans-Peter; und Monika (meist mit Zusatznamen), Renate, Elke, Birgit, Brigitte, Ursula, Christel, Erika, Astrid, Sigrid, Doris. Es kommen auch Namen vor wie: Alfred, Arnold, Dietwin, Ewald, Gerhard, Gerold, Hagen, Heinrich, Helmut, Herbert, Holger,

${ }^{11}$ Eine Harmonie, die bei Frauen, die durch die Heirat den Nachnamen ihres Mannes annahmen, verlorengehen konnte. 
Horst, Karl-Heinz, Kurt, Manfred, Ralf, Reinhard, Reinhold, Rolf, Rudolf, Siegfried, Ulf, Volkmar, Walter, Wilhelm, Wolfgang - eindeutig alte deutsche Namen. Dazu sind immer noch auch die eingedeutschten biblischen und Heiligennamen präsent Andreas, Georg (Jörg), Johann (Hans), Michael, Thomas - ohne aber zu den häufigsten Namen zu gehören. Bei den Mädchen trifft man reichlich auf alte deutsche Namen wie: Gertrud, Gudrun, Hedda, Heidrun, Hilda, Hildegard, Sunhilde, Ulrike - und auch die Klassiker sind (meist als Zweitname) vertreten: Elisabeth, Maria, Susanna. Einzig nicht explizit deutsch klingende Namen, mit jeweils nur einer Nennung, sind: Isabela, Patricia, Corinna, Gabriela, Iris.

Zum Vergleich ${ }^{12}$ : Beliebteste Jungenvornamen in Deutschland im Jahr 1970: „Stefan, Michael, Andreas, Thomas, Frank, Markus, Christian, Oliver, Mathias, Torsten." 13 (Die Namen Oliver und Mathias tauchen in Hermannstadt in dem Jahr überhaupt nicht auf, Torsten mit einer Nennung.) Im Jahr 1970 herrschen in Deutschland keine germanischen Namen vor, sondern hauptsächlich die christlichen eingedeutschten Namen. Und bei den Mädchen: „Nicole, Anja, Claudia, Stefanie, Andrea, Tanja, Katrin, Susanne, Petra, Sabine." ${ }^{14}$ Die Top-Namen Nicole, Anja, Stefanie und Tanja kommen in dem Stichjahr in Hermannstadt überhaupt nicht vor, während Petra, Sabine und Susanne auch hier Nennungen haben. Der in Deutschland in

${ }^{12}$ Es handelt sich hierbei um zwei sehr unterschiedliche Größen: einerseits die Hitliste für ganz Deutschland, andererseits das Namensrepertoire einer kleinen Gemeinschaft in Siebenbürgen. 1970 zählte die Hermannstädter evangelische Gemeinde etwa 15.000 Mitglieder und es gab 363 Taufen. Bis auf nur drei Ausnahmen wurden Kinder getauft, die erst wenige Monate alt waren. Die Namensgebung und die Taufe liegen demnach zeitlich nah beieinander, was für die Namensforschung wichtig ist.

${ }^{13}$ Bielefeld, Knud: Beliebte Vornamen. Hitlisten. Abgerufen von: https://www.beliebte-vornamen.de/hitlisten [10.11.2018].

${ }^{14}$ Ebenda. 
den 1970ern modische Name Claudia kommt hier nur zweimal, und zwar in deutsch-rumänischen Familien, vor.

Der Hermannstädter Spitzenreiter-Name Monika kommt oft in Kombination mit einem zweiten Namen vor: MonikaRenate, Monika-Ute, Monika-Gerlinde, Heidrun-Monika, Karin-Monika, Monika-Adelheid, Ruhtraut-Monika. Die Wahl des zusätzlichen Namens markiert die ethnisch-deutsche Identität.

\section{Hybridisierung: Namenspräferenzen multilingualer Familien}

Die Interkulturalitätsforscherin Helene Haas legte 2009 eine grundlegende Studie, Das interkulturelle Paradigma, vor, in der sie mit großer Genauigkeit alle Lehrmeinungen zum Thema Interkulturalität untersucht. Unter vielen anderen vorgestellten Meinungen dokumentiert sie:

Bolten gibt zu bedenken, dass jede Kultur auch durch Interaktion mit anderen Gruppierungen geprägt ist und kollektive Wissensvorräte nicht mehr klar voneinander getrennt sind. Hinnenkamp zufolge kann man „kaum mit Bestimmtheit sagen, wo eine Kultur aufhört und eine andere anfängt". ${ }^{15}$

Und sie schlussfolgert: „Möchte die Interkulturelle Kommunikation am Puls der Zeit bleiben, muss sie der kulturellen Hybridität Rechnung tragen." ${ }^{\text {"16 }}$ Hybridität meint die Vermischung von Kulturen und die dadurch bedingte Entstehung von etwas Neuem. Möchte man weniger von kulturellen Abgrenzungen als von dem Gemeinsamen sprechen, so ist das Konzept der Multikollektivität passend: die gleichzeitige Zugehörigkeit einer Person zu mehreren Kollektiven. In Siebenbürgen machen Kinder aus gemischtsprachigen und gemischtkonfessionellen

${ }^{15}$ Haas, Helene: Das interkulturelle Paradigma. Passau: Verlag Karl Stutz, 2009, S. 129.

${ }^{16}$ Ebenda. 
Familien diese Erfahrung der Multikollektivität. Manchmal ist es auch eine negative Erfahrung, nämlich die, zwischen den Stühlen zu sitzen und zu keinem der beiden Kollektive - weder zur sächsischen Gesellschaft, noch zum rumänischen Sozialgefüge - richtig dazuzugehören. Am Namen, den sie tragen, sollte es jedoch nicht liegen, denn die transkulturellen, internationalen Namen, die die meisten gemischtsprachigen Elternpaare fürsorglich ausgewählt haben, sollten wunschgemäß in beide Milieus passen.

Vereinfacht gilt für diese Untersuchung: hat ein Elternteil einen deutschen Familiennamen und ist evangelischer Kirchenzugehörigkeit und das andere Elternteil hat einen rumänischen Familiennamen und ist zugleich Angehöriger der rumänischorthodoxen Kirche, so gilt die Familie als deutsch-rumänische Familie.

These 3: Gemischtsprachige Familien wählen Namen, die in beiden Sprachen üblich sind.

Statistische Daten zu Vornamen in deutsch-rumänischen Familien, 1970: Im Jahr 1970 gab es in der Hermannstädter Evangelischen Kirchengemeinde 363 Taufen. In 60 Fällen (16 Prozent) war ein Elternteil nicht evangelisch A. B., sondern rumänisch-orthodox (und zugleich mit rumänischen Namen), reformiert (und zugleich mit ungarischem Namen), römischkatholisch (mit deutschem oder ungarischem Namen) oder freikirchlich-evangeliumschristlich (deutsch).

Die Kinder aus den gemischtkonfessionellen und deutschrumänisch gemischtsprachigen Elternhäusern des Jahres 1970 heißen, mit Mehrfachnennungen:Adrian, Alexander, Christian ${ }^{17}$, Emil, Robert, Alexandra, Claudia, Laura; und mit

${ }^{17}$ Die Namen Christian (häufig), Richard, Roland, Werner, Andreas, Alexandra kommen auch in endogamen deutschsprachigen Familien vor, wobei in diesen Fällen Christian oft als Doppelname mit einem deutschen Namen kombiniert wird: Christian-Bernhard, -Michael, -Kurt, -Klaus, Hans-Christian u. a. 
Einzelnennungen: Monika, Christina, Carmen, Daniela, Maria, Betina/Bettine, Valeria, Andreas, Andrea, Mircea, Harry/Harri, Demora, Karla, Claus, Eduard, Marian, Oskar, Nikolaus, Carina, Bianca, Corina, Cornelia, Mihaela, Martina, Norbert, Adonis, Richard, Roland, Werner (nach dem Vater), Stefan, Eugen, Viktor, Ferdinand.

Die Namen der Kinder aus deutsch-ungarischen Familien sind: Zoltan, Enikö, Eveline, Evelyn, Harriet, Anita, Antonia, Carina, Sybille, Edith, Arpad-Gerhard, Alexander, Arthur, Judith. Das ungarische Element möchte sichtbar zur Geltung kommen, während die Anpassung an den deutschen oder rumänischen Namenschatz fallweise ebenfalls zu erkennen ist.

\section{Diversifizierung: Annäherung an transkulturelle europäische Trends}

Auf dem Rufnamen „ballt sich (...) ein Konglomerat aus Alter, Nationalität, Regionalität, Ethnizität, Geschlecht und manchmal auch Religion bzw. Konfession" ${ }^{\text {"18, schreibt Damaris }}$ Nübling. Nicht immer und nicht für alle Hörer des Namens erschließen sich diese Informationen. Je internationaler die Namen werden, umso schwieriger wird es, Personen nach ihrem Namen den genannten Kategorien zuzuordnen.

Sieht man sich die Listen der beliebtesten Namen in Deutschland und in anderen europäischen Ländern an, werden einige Trends erkennbar und zwar: Heute suchen Eltern sowohl für Mädchen als auch für Jungen klangvolle, weiche, kurze Namen aus wie Ben, Fynn, Leo, Mia, Lisa, Emma. Die Motive der Namenswahl haben sich geändert. Mögliche Beweggründe bei der Namensvergabe fasst der Psychologe Udo Rudolph folgendermaßen zusammen:

Müller (2001) nimmt an, dass gegenüber früheren Gepflogenheiten

- die eigenen Kinder beispielsweise nach Familientraditionen,

${ }^{18}$ Nübling, 2012, S. 140. 
lokalen Heiligen oder den Regenten des Landes zu benennen - heutzutage so genannte „subjektive“ Merkmale wie Modernität, Klang und Länge des Namens eine viel größere Rolle bei der Namensvergabe spielen. EineUmfrage im Auftrag des Wilfried-Seibicke-Instituts für Namensforschung ergab 1999 einen Anteil subjektiver Beweggründe bei der Namensvergabe von $68 \%$; im Gegensatz zu nur 17\% der befragten Eltern, die sich an (Familien-) Traditionen orientiert hatten. ${ }^{19}$

These 4: Auch in Siebenbürgen wählen Eltern Namen, die in Europa gerade ,in“ sind.

Statistische Daten zu evangelischen Taufnamen 2000: Im Jahr 2000 gab es in der Hermannstädter evangelischen Gemeinde sieben Taufen. Von den Elternpaaren waren sechs gemischtkonfessionell. Die drastisch gesunkene Zahl der Taufen deutet nicht auf eine Entkirchlichung hin, sondern sie ist die direkte Folge der massiven Auswanderung der Gemeindemitglieder im Zuge des Exodus der Siebenbürger Sachsen Anfang der 1990er Jahre. Der in Hermannstadt von evangelischen Eltern im Jahr 2000 vergebene Namen Laura steht in Deutschland in demselben Jahr in der Top-10-Liste. Der Name Christian war einige Jahre zuvor im Top, ebenso Patrick. Der Name Evelyn/Evelin wurde in Hermannstadt zweimal vergeben.

Statistische Daten zu evangelischen Taufnamen 2017: Im Jahr 2017 gab es in der Hermannstädter evangelischen Gemeinde 13 Taufen. Von den Elternpaaren sind neun gemischtkonfessionell. Mindestens drei Paare haben den Wohnsitz außerhalb Rumäniens, ließen aber die Kinder hier taufen.

Einige der 2017 vergebenen Namen gehören zu den beliebtesten Namen desselben Jahres in Deutschland, so die Namen

${ }^{19}$ Rudolph, Udo; Böhm, Robert; Lummer, Michaela: Ein Vorname sagt mehr als 1000 Worte. Zur sozialen Wahrnehmung von Vornamen. Zeitschrift für Sozialpsychologie, 38 (2007) S. 17-31. (DOI:10.1024/ 0044-3514.38.1.17.) Abgerufen von https:/www.researchgate.net/ publication/236876507_Ein_Vorname_sagt_mehr_als_1000_Worte Zur_sozialen_Wahrnehmung_von_Vornamen [11.11.2018]. 
Felix, Lukas, Maya. ${ }^{20}$ Weitere, ebenfalls in Hermannstadt vergebene Namen - Marcel, Jacob, Johanna, Leonora, Victor gehören auch zu den häufigen Namen der Gegenwart. Der weich und feminin klingende Namen Evelyne/Evelin wurde wieder zweimal vergeben.

Sowohl im Jahr 2000, als auch 2017 wählten die evangelischen und die gemischtkonfessionellen Elternpaare keine germanischen Namen für ihre Kinder, sondern Namen, die sowohl deutsch als auch rumänischgängig sind, wie Laura, Robert, Victor, - ggf. mit erkennbar deutscher Form: Markus, Andreas, Johanna. Etwa die Hälfte der Kinder trägt zwei Vornamen. Ein Trend zu besonders kurzen Namen (vom Typ Mia u. Leo) ist nicht zu erkennen, wohl aber ein Bestreben nach Sonorität (Evelyne, Leonora, Serina, Milan). Zwei Kinder hatten als zweiten Vornamen einen rumänischen bzw. ungarischen Namen.

Fazit: Nach 1990 ist die beobachtete Gruppe zu klein, um fundierte Schlüsse ziehen zu können. Die regionale deutschsprachige Gemeinschaft ist im Verschwinden begriffen. Es kann jedoch behauptet werden, dass durch das Zusammenleben der unterschiedlichen Ethnien in Hermannstadt bei der Namengebung deutsch-rumänische Interferenzerscheinungen auftreten. Demnach - und zugleich als Folge der Globalisierung - ist auch in diesen Breiten eine zunehmende Diversifizierung und Interkulturalität in der Namenwahl festzustellen.

${ }^{20}$ Die beliebtesten Namen für Neugeborene in Deutschland waren im Jahr 2017, laut Gesellschaft für die deutsche Sprache: Maximilian, Alexander, Paul, Elias, Ben, Noah, Leon, Louis/Luis, Jonas, Felix; sowie: Marie, Sophie/Sofie, Maria, Sophia/Sofia, Emilia, Emma, Hannah/Hanna, Anna, Mia, Luisa/Louisa. Quelle: Gesellschaft für deutsche Sprache e. V.: Die beliebtesten Vornamen. Abgerufen von: https://gfds.de/vornamen/beliebteste-vornamen/ [10.11.2018]. Knud Bielefeld kommt zu ähnlichen Resultaten, da führen Ben und Emma die Liste an. 


\section{Literaturverzeichnis}

\section{Primärquellen}

Taufregister der Evangelischen Kirchengemeinde A.B. Hermannstadt, Zentralarchiv der Evangelischen Kirche A.B. in Rumänien. Pfarramtliche Matrikelabschriften der Jahrgänge 1910, 1940, 1970. Bestand ZAEKR: 400/276.

Taufregister der Evangelischen Kirchengemeinde A. B. Hermannstadt, Matrikeln der Jahrgänge 2000 und 2017. (In Form von Mitteilung des Pfarramtes unter Wahrung der Personendatenschutzbestimmungen.)

Weber, Georg; Weber, Renate: Zendersch. Eine siebenbürgische Gemeinde im Wandel. München: Delp, 1985.

\section{Sekundärliteratur/Fachliteratur}

Nübling, Damaris: Namen. Eine Einführung in die Onomastik. Tübingen: Narr, 2012.

Haas, Helene: Das interkulturelle Paradigma. Passau: Verlag Karl Stutz, 2009.

Heringer, Hans Jürgen: Interkulturelle Kommunikation. 4. Aufl. Tübingen: A. Francke Verlag, 2014.

Löffler, Heinrich: Germanistische Soziolinguistik. 4., neu bearb. Aufl. Berlin: Erich Schmidt Verlag, 2010.

\section{Internetquellen}

Bielefeld, Knud: Beliebte Vornamen. Hitlisten. Abgerufen von: https://www.beliebte-vornamen.de/hitlisten [10.11.2018]. Gesellschaft für deutsche Sprache e. V.: Die beliebtesten Vornamen. Abgerufen von:https://gfds.de/vornamen/beliebteste-vornamen/ [10.11.2018].

Rudolph, Udo; Böhm, Robert; Lummer, Michaela: Ein Vorname sagt mehr als 1000 Worte. Zur sozialen Wahrnehmung von Vornamen. Zeitschrift für Sozialpsychologie, 38 (2007) S. 17-31. (DOI:10.1024/0044-3514.38.1.17.) 
Abgerufen von https:/www.researchgate.net/publication/236876507_Ein_Vorname_sagt_mehr_als_1000_ Worte_Zur_sozialen_Wahrnehmung_von_Vornamen [11.11.2018].

\section{Weiterführende Literatur}

Busch, Brigitta: Mehrsprachigkeit. Wien: facultas.wuv, 2013. DUDEN. Das große Vornamenlexikon. 2. völlig neu bearb. Aufl., bearb. von Kohlheim, Rosa; Kohlheim, Volker. Mannheim: Bibliographisches Institut - Dudenverlag, 2003.

Drosdowski, Günther: Lexikon der Vornamen. Herkunft, Bedeutung und Gebrauch von mehreren tausend Vornamen. 2., neu bearb. und erw. Aufl. Mannheim: Bibliographisches Institut - Dudenverlag, 1974.

Kuße, Holger: Kulturwissenschaftliche Linguistik. Eine Einführung. Göttingen: Vandenhoeck \& Ruprecht, 2012. McArthur, Marilyn: Zum Identitätswandel der Siebenbürger Sachsen. Eine kulturanthropologische Studie. (G. Weber, Hrsg. \& U. Maurer, Übers.) Köln, Wien: Böhlau, 1990. 